\title{
Reseña del coloquio Pensar la Historia del Tiempo Presente, Universidad Iberoamericana (22-26 de febrero de 2016)
}

\author{
Margarita Pérez Caballero* \\ Centro de Investigación y de Estudios Avanzados \\ margaritaperc@yahoo.com.mx
}

Historia del tiempo presente o del presente; historia reciente o de nuestro tiempo; historia del mundo actual o historia próxima o inmediata son algunas de las formas empleadas para referir un modelo historiográfico cuya temporalidad está íntimamente relacionada con la coetaneidad; es decir, con una "historia vivida" que conforma la historicidad "según es percibida por los sujetos que actúan en una determinada coyuntura temporal": es "la historia que perciben los propios sujetos que la viven" (Arostegui, s.f.: 1). Hacer referencia a esta subdisciplina es cuestionar la herencia positivista según la cual la distancia temporal entre los acontecimientos que van a historiarse y el historiador debe ser contundente. Sin embargo ¿en qué año acaba la historia? ¿A partir de qué fecha debe enmudecer el historiador? (Soto, 2004: 102). La historia del tiempo presente, modelo historiográfico que desafía el "enmudecimiento" del historiador, se gestó en la segunda mitad del siglo Xx con fundamentos epistemológicos y metodológicos que se han ido consolidando en las últimas dos décadas. "Hoy, más que un 'concepto en construcción' es un concepto que requiere de su aplicación [...] dar cuenta en forma 'práctica' de lo que estamos propugnando" (Soto, 2004: 102).

Lo anterior fue discutido a profundidad durante el primer coloquio organizado por el Departamento de Historia de la Universidad Iberoamericana: Pensar la Historia del Tiempo Presente. Mediante ocho mesas de exposición y análisis, desarrolladas a lo largo de cinco días, conferencistas y ponentes pusieron de relieve el rumbo que lleva la subdisciplina en México, con ejemplos de proyectos de investigación de diversa índole. La amplitud del encuentro hace necesario dividir esta reseña en dos apartados: uno para la presentación de los aspectos teórico-metodológicos discutidos, y otro para las temáticas en investigación. Finalmente, concluyo con algunas reflexiones para el debate.

\footnotetext{
* Estudiante de doctorado del Departamento de Investigaciones Educativas, Cinvestav.
} 


\section{Tiempo y memoria: aspectos teórico-metodológicos}

Desde su nacimiento, la historia del tiempo presente se ha considerado una visión europeísta de la realidad, y ha recibido diferentes denominaciones: hacia 1978, en una publicación dirigida por Jacques LeGoff se conceptualiza el término "historia inmediata"; en 1979 se funda en Francia el Institut d’Histoire du Temps Présent, y Julio Aróstegui habla, desde 1989, sobre la "historia vivida". Las motivaciones para la adopción de una u otra denominación son diversas y, en algunos casos, su empleo está asociado al estudio de problemáticas específicas. En el contexto latinoamericano, en el Cono Sur se emplea la expresión historia del pasado reciente para abordar el periodo de las dictaduras militares de las décadas de 1960 y 1970; historia coetánea es un término poco difundido debido a que no existe en todas las lenguas; y en el caso de México, el campo se está perfilando bajo la denominación historia del tiempo presente. Dar cuenta de este debate fue parte de lo expuesto por Eugenia Allier, del Instituto de Investigaciones Sociales de la Universidad Nacional Autónoma de México (UNAM).

Respecto a la discusión entre las denominaciones historia contemporánea e historia del tiempo presente, Ilán Semo, de la Universidad Iberoamericana, argumentó sobre una relación específica de este presente con el pasado y futuro, debido a que el pasado se divide cada vez en más periodos y estos periodos son también cada vez más cortos. Desde este enfoque, dijo, la modificación del tiempo histórico ha generado un cierre de futuro. Es decir, dejan de verse cambios históricos posibles, detenidos, al pasar de un horizonte utópico generado en la década de 1960, a un horizonte distópico provocado por el colapso de expectativas de la década de 1980. Por ello, a diferencia de historia contemporánea, la denominación historia del tiempo presente se refiere no a un corte temporal específico, sino a un cambio de régimen de historicidad; una nueva manera de percibir el tiempo, donde el tiempo presente se emplea como base de una historia con otros problemas que se van a abordar. Este aspecto suscitó en el coloquio interesantes planteamientos respecto del tiempo.

Así, Silvia Pappe, de la Universidad Autónoma Metropolitana, unidad Azcapotzalco, considera la temporalidad como un tema no resuelto en su totalidad por el historiador: "hay cosas que no sabemos del tiempo", pero lo que sí sabemos es que al ser lo moderno tan transitorio, fugaz y contingente, el presente continuo pierde temporalidad y la proyección que se hace hacia el futuro es cada vez más incierta; sin embargo, sigue siendo presente, y para observar este presente es necesario llevar a cabo una representación de nuestro conocimiento sobre las cosas, lo cual nos lleva a una observación en segundo grado, es decir, el que observa es parte de lo que está observando y, en este sentido, en el historiador del tiempo presente se refuerza la necesidad de construir un relato como conocimiento. 
Para recapacitar sobre los planteamientos de Pappe cabe retomar la noción moderna de tiempo presentada por William Brinkman, de la Universidad Iberoamericana: el tiempo ha dejado de ser una línea curva que delimita el propio tiempo, para ser una línea recta que atraviesa el mundo, lo cual coloca al presente como instante cero. Eufemio Franco, investigador independiente, considera que el espacio, entonces, comparte un punto con el tiempo, lo cual hace que los acontecimientos se relacionen en más de un modo. De ahí que, donde el ser es, el tiempo no está presente. También recalcó: el presente nunca cambia, el pasado y el futuro están supeditados a éste y, en el entendido de que el tiempo cíclico se trastoca en presente, es necesario manejar espacio y tiempo en una visión histórica actual. Lo anterior puede derivar en una historia del tiempo presente conceptualizada como una forma de observación, al hacer del tiempo una condición de posibilidad para todo objeto de estudio, señaló Fernando Betancourt del Instituto de Investigaciones Históricas (UNAM). Esta historia se desplaza conforme transcurre el tiempo, sobre todo si consideramos que el futuro sólo existe porque el presente se convierte en pasado, afirmó Rodrigo Laguarda del Instituto Mora.

Por otra parte, para Eugenia Allier la memoria es la matriz y objeto de esta historia, y su irrupción se ve reflejada en los movimientos estudiantiles de la década de 1960. La cercanía a tales acontecimientos, planteó Daniel Ovalle, de la Universidad de Chile, ubica al presentismo y la memoria como conceptos-vectores de la historia del tiempo presente. Con ello hizo referencia a la noción de presentismo de François Hartog, régimen de historicidad de nuestros días caracterizado por el dominio de la categoría del tiempo presente, donde el futuro aparece cerrado. Por lo tanto, como afirmó Alejandro Cheirif, del Centro de Investigación y Docencia Económicas, el presentismo constituye una herramienta heurística para el análisis de archivos.

En cuanto a la naturaleza de la memoria, esta hace referencia a percepciones sobre un mismo acontecimiento y, por ende, a la coexistencia de diferentes pasados-presentes en pugna por la verdad, lo cual hace necesario abordar la memoria desde dos planos: el individual y el colectivo. Explicar y comprender ambos planos permite identificar acciones no percibidas por la memoria individual y sí por la colectiva. Esto llevaría, según Margarita Olvera, de la Universidad Autónoma Metropolitana, Unidad Azcapotzalco, a observar la memoria como construcción de primer grado y a la historia como construcción de segundo grado.

En el coloquio no faltaron las alusiones a los grandes teóricos del tiempo histórico y de la memoria, como Koselleck, Hobsbawm, Ricoeur y Todorov, entre otros. Pero más allá de la discusión teórica de los aspectos constitutivos de la historia del tiempo presente, también se presentaron trabajos que usan esa perspectiva para el estudio de otras temáticas. De esos trabajos se hace un recuento en el siguiente apartado. 


\section{De la teoría a la práctica: actores, instituciones y temáticas}

Aunque la discusión de las categorías de tiempo y memoria fue central en el coloquio, un buen número de trabajos se dedicó a mostrar cómo pueden ponerse en práctica los métodos y herramientas de la historia del tiempo presente. Investigadores adscritos a distintas instituciones, ${ }^{1}$ así como investigadores independientes, ${ }^{2}$ compartieron interesantes propuestas en cuanto al rescate de evidencia empírica, la aplicación de encuestas a actores clave y la búsqueda de testimonios de la "historia vivida". Se rebasaría la extensión de esta reseña al tratar de enumerar todos y cada uno de los trabajos presentados, por lo que intentaré salvar la circunstancia por medio de la enunciación de temáticas generales y lo más sobresaliente de ellas.

Las investigaciones sobre política y violencia siguen siendo dominantes en la historia del tiempo presente, como lo demuestran las presentaciones siguientes: "Las fuentes de la memoria: la construcción de narrativas sobre el pasado reciente en el caso del Proyecto Colombia Nunca Más", relacionado con crímenes de lesa humanidad cometidos de 1966 en adelante. La ponencia "¿Es posible hacer una historia de la violencia? Aproximaciones desde la historia del tiempo presente" puso sobre la mesa un cuestionamiento historiográfico. La guerrilla en México fue abordada en "Tramitación del pasado y reconfiguraciones de la memoria. Ex guerrilleros de los setenta ante la 'transición democrática' en México. 1965-2000”. La mirada de un embajador fue estudiada en "Diplomacia en tiempos de guerra: Gustavo Iruegas en Nicaragua y El Salvador, 1978-1981", basada en entrevistas con el propio Iruegas. Los derechos humanos fueron el centro de "Historiando los derechos en México. Historias de vida de los defensores". Un poco diferente, con reflexiones sobre maneras distintas de concebir el tiempo, fue la ponencia "Políticas de la conmemoración y regímenes de historicidad: dos entradas conceptuales para la historización de los bicentenarios de Independencia (México y Colombia, 2010)". También se abordaron el arraigo y el desarraigo como elementos para la creación de una nueva acepción de memoria, ya sea comunicada o cultural, y sus efectos sociales en "La experiencia de la temporalidad histórica en las memorias rotas de los exiliados: hacia nuevos relatos en tiempo presente", y la construcción de una memoria sin

\footnotetext{
${ }^{1}$ Universidad Iberoamericana; Universidad Nacional Autónoma de México; Universidad de Chile; Centro de Investigación y Docencia Económicas; Universidad Autónoma de Puebla; Universidad Autónoma Metropolitana, unidades Azcapotzalco, Cuajimalpa, Iztapalapa y Xochimilco; Universidad de Guanajuato; Escuela de Estudios Superiores en Ciencias Sociales-París; 17 Instituto de Estudios Críticos; Universidad de Guadalajara; Departamento de Investigaciones Educativas (Cinvestav); Escuela Nacional de Antropología e Historia; Instituto Mora; Durham University; Universidad Autónoma de Querétaro, y El Colegio de Michoacán.

2 Eufemio Franco y Clara Bolívar.
} 
proponérselo en "El estudio del movimiento social de ex braceros desde la historia del tiempo presente", acontecido durante el gobierno del presidente Vicente Fox, entre otros.

Hubo dos presentaciones de temática indígena: "Temporalidad compleja en el canto ritual wixárika", investigación relacionada con los rituales ancestrales llevados a cabo por el pueblo huichol, y "Regímenes de historicidad y formación nacional de alteridad: 'Lo indígena' en México". En cuanto a educación, dos estudios fueron presentados con miras al rescate de las memorias docentes: "Tita, 'mi vocación fue la educación'. Experiencias con la memoria y la enseñanza"; y "Memorias de un proceso en la historia del tiempo presente: Equipos Docentes de México (1960-1980)", una asociación de docentes de fuerte arraigo religioso que laboran en todos los niveles de la educación pública. Y tres trabajos de historia de la vida cotidiana "La Merced: el barrio de La Soledad", "Los santos como espejo de la sociedad", y "Rescatando un pasado: entre memorias y relatos del sismo del 85 ".

El tema del arte fue convocado a través de varias disciplinas, una de ellas el performance: "Marina Abramovic: Performance en el presente", descrito como la relación directa entre la obra creada en el pasado y la puesta en escena del presente, que trata de explicar y hacer confluir estos dos momentos. En el teatro, "Memoria y canon: la antología del teatro mexicano del siglo Xx". El arte arquitectónico fue abordado en "Monumento, documento: el patrimonio cultural como representación historiográfica de la memoria". Y de tema literario fue "Las pesadillas sin testigo. Olvido y narración en Jorge Luis Borges".

Entre las ponencias con propuestas metodológicas novedosas figuran: "Armando un método de investigación histórica: el paisaje de la microcuenca maconí, Cadereita de Montes", "De-lete: Olvido, registros audiovisuales cotidianos y nuevas tecnologías de archivación" y "El periodismo de investigación como referente para pensar la historia del tiempo presente". Otros modelos historiográficos se fusionaron con la historia del tiempo presente para dar paso a "híbridos" por demás interesantes. Por ejemplo, la historia conceptual fue empleada para rastrear el concepto de Estado-nación a través del perfil biográfico de Hanna Arendt. La historia de las emociones condujo a trabajos como "De la emoción al nombre: seudónimos en la comunidad femenina de El Hogar", "Ausencias y dramas. Tras la huella visual del dolor", "La guerra nazi contra los homosexuales. Una mirada al trauma de las minorías europeas del siglo xx" y "La fotografía como relato afectivo. La nación sentida".

Finalmente, en el marco del coloquio se llevó a cabo la presentación del libro La Liga Comunista 23 de Septiembre. Cuatro décadas a debate: historia, memoria, testimonio y literatura, coordinado por Rodolfo Gamiño, Yllich Escamilla, Rigoberto Reyes y Fabián Campos, y editado por el Programa de Posgrado en Estudios Latinoamericanos de la UnAM y la Facultad de Ciencias para el Desarrollo Humano de la Universidad Autónoma de Tlaxcala. 


\section{Reflexiones para el debate}

Martín López Ávalos, de El Colegio de Michoacán, plantea que la historia de tiempo cerrado es un saber canonizado. Hacer historia del tiempo presente es hacer un ejercicio de tiempo abierto, que genera tensión en el canon establecido, donde uno de los principales problemas es el de la inmediatez. Lo anterior problematiza sobremanera el uso de las fuentes, que pierden legitimidad "en sí mismas" y ello obliga al historiador a construir fuentes y validarlas. Como señaló Rodrigo Laguarda, del Instituto Mora, a menor distancia temporal del acontecimiento hay un compromiso mayor del historiador, debido no a la escasez de fuentes sino, al contrario, al riesgo de reproducir discursos acríticos ante la vorágine de la información; es el riesgo de repetir consignas en lugar de construirlas. Para ello es necesario especificar cuál es el presente de las sociedades en estudio, tomar en cuenta su pertinencia social, y considerar la interdisciplinariedad como práctica científica validada. El trabajo ya no puede ser individual, advirtió Martín López, pues se hace inevitable el traslape entre los estudios de los antropólogos, los sociólogos y los historiadores.

Ante estas circunstancias, señalaron María del Carmen Collado y Héctor Zarauz, del Instituto Mora, se vislumbran nuevos retos para el historiador que cohabita con los acontecimientos: "asumir su carácter de observador participante como narrador", "hablar de la política con más responsabilidad que compromiso" y "en aras de la pasión por el conocimiento presente, correr el riesgo de desmitificar al sujeto de estudio".

Del papel que juega la memoria en la construcción de estudios relacionados con el tiempo presente puede decirse, con Eugenia Allier, que "la memoria, la historia y el presente son una tríada amarrada por la política". En efecto, la historia del tiempo presente nació ligada a la violencia y la política; sin embargo, en la presentación de trabajos de investigación del coloquio no se observa un estancamiento en esa temática estricta. Si bien la vuelta de tuerca ha sido lenta se perfilan nuevos objetos de estudio, como las investigaciones de carácter educativo no vinculadas a los movimientos estudiantiles.

¿Cómo incide esta perspectiva y todas sus implicaciones en la investigación educativa? La aldea global está modificando sus prácticas y una de ellas es la relacionada con la educación. Los cambios en los modelos educativos; el uso de la tecnología que impulsa el despliegue de formas no escolarizadas de enseñanza; y la movilidad mundial que provoca prácticas culturales, también con importante movilidad, hacen necesario volver la mirada al presente educativo ¿Cómo hemos transitado del pasado reciente al presente inmediato? ¿Cuáles son las prácticas, en materia educativa y cultural, que habrán de mantenerse en el olvido? ¿Cuáles memorias será necesario preservar para transmitir integeneracionalmente y dar así continuidad a la "historia vivida"? Podrá argumentarse que estos cuestionamientos también serían aplicables a un tiempo histórico cerrado, pero el dinamismo de los cambios 
hace cada vez más escasas las continuidades. En consecuencia, no considerar de manera objetiva la aceleración física del universo y, subjetivamente, el propio sentimiento de aceleración de la historia, implica paradójicamente la fragmentación, la falta de continuidad en nuestras vidas históricas, en términos educativos.

\section{Fuentes}

Arostegui, Julio (s.f.). "El tiempo presente como tema de investigación histórica y como problema didáctico", documento pdf, disponible en: <www.fedicaria.org/miembros/nebraska/jaca07/1_AROSTECUI.pdf>, (fecha de consulta: 8/10/2014).

González, Magdalena (2015), "La teorización de Julio Aróstegui sobre la historia del tiempo presente como historia vivida", Hispania Nova, núm. 13, pp. 126-133, documento pdf, disponible en: <www. uc3m.es/hispanianova>, (fecha de consulta 18/4/2016).

Le Goff, Jacques, Royer Chartier y Jacques Revel (1978), La Nouvelle histoire, Retz, París.

Soto, Ángel (2004), "Historia del presente: estado de la cuestión y conceptualización", Historia Actual Online, núm. 3, documento pdf, disponible en: <www.historia-actual.org/Publicaciones/index.php/ haol/article/viewFile/34/35>, (fecha de consulta 8/10/2014). 